\title{
Interacting Vortices and Spin-up in 2-D Turbulence
}

\author{
J. B. Taylor*, Matthias Borchardt**, Per Helander** \\ * Radwinter, Wallingford, OX10 9EJ, UK \\ ** Max Planck Institute for Plasma Physics, 17491 Greifswald, \\ Germany
}

\begin{abstract}
In simulations of decaying two-dimensional turbulence, Clercx et al $[1,2]$ discovered the unexpected phenomenon of "spin-up". (This is the spontaneous acquisition of angular momentum by a turbulent 2-D fluid in a rigid container.) Here we show that this phenomenon can readily be interpreted in terms of statistical models of two-dimensional turbulence. When the net vorticity is zero in a bounded system there are two distinct types of statistical equilibrium. The first has the expected property that its angular momentum is zero. However, the second type has non-zero angular momentum even though its circulation vanishes. The relative probability of the two types of equilibrium depends strongly on the shape of the boundary and weakly on the energy. The angular momentum predicted for the second type of equilibrium is in good agreement with that found in simulations at high Reynold's number.
\end{abstract}

Two-dimensional turbulence, (which may to some extent be realized in magnetized plasmas, in rotating and stratified fluids, and in thin films), has been extensively studied by direct numerical simulation. In high Reynolds number simulations of decaying 2-D turbulence, Clercx et al [1,2] discovered the remarkable phenomenon of "spin-up". This is the spontaneous acquisition of angular momentum by a turbulent 2-D fluid in a rigid container - a process that depends on the shape of the container.

It has often been proposed $[3,4,5,6]$, originally by Onsager [7], that 2-D turbulence might be interpreted in terms of the statistical equilibria of interacting point vortices or equivalently of interacting charged rods in a parallel magnetic field $[8,9,10]$. Here we show that this, and other statistical models, readily account for spin-up in a bounded domain - both qualitatively in its dependence on boundary shape and quantitatively in the amount of angular momentum it generates. 
The motion of an incompressible inviscid fluid in 2-D can be described by a stream function $\psi(x, y)$ and the $z$-component of vorticity $\omega(x, y)$ through the "Euler Eqs":

$$
\begin{gathered}
\frac{\partial \omega}{\partial t}+u \frac{\partial \omega}{\partial x}+v \frac{\partial \omega}{\partial y}=0, \\
u=\frac{\partial \psi}{\partial y}, \quad v=-\frac{\partial \psi}{\partial x}, \\
\nabla^{2} \psi+\omega=0 .
\end{gathered}
$$

If the continuous vorticity is represented by a distribution of discrete line-vortices (points in 2-D), their equations of motion are[7]

$$
e_{i} \frac{d x_{i}}{d t}=\frac{\partial H}{\partial y_{i}}, \quad e_{i} \frac{d y_{i}}{d t}=-\frac{\partial H}{\partial x_{i}}
$$

where $e_{i}$ is the strength of each vortex. The Hamiltonian is the interaction energy [11] $\sum e_{i} \psi\left(x_{i}, y_{i}\right)=\sum e_{i} e_{j} K\left(x_{i}, y_{i}: x_{j}, y_{j}\right)$ and $K$ is the Green's function for the Laplace operator with appropriate boundary conditions.

These equations also describe the motion of charged rods $[8,9,10]$ aligned with a magnetic field and moving with velocity $\mathbf{E} \times \mathbf{B} / B^{2}$ - when the Hamiltonian is the electrostatic potential $\phi(x, y)$ of the rods. This easily visualized mechanical analogy supports the application of statistical mechanics to the point vortex model and hence to fluid turbulence.

A more formal method of introducing statistics into 2-D turbulence was described by Robert and Sommeria [12]. This dispenses with a mechanical model: instead it breaks up the vorticity into small "patches" and distributes these statistically to find a most probable state, in the manner introduced by Lynden-Bell [13]. In principle this approach enables one to incorporate all the invariants of the Euler Eqs (1-3). However, the results then depend on the detailed initial conditions and in practice, because of its complexity, this approach has been used only for simple initial distributions [14, 15] or, as in [12], in a linear approximation - where it is equivalent to the point-vortex model (see Eq (7) below).

It is not clear that all the invariants of the ideal Euler Eqs should be applied to a fluid with a small viscosity, and we are interested only in generic properties, independent of detailed initial conditions. We therefore follow many authors $[3,4,5,6,7,8,9,10,11]$ and represent the fluid by a distribution of point vortices with a fixed total energy $E$. We consider only the case of zero total vorticity, (when spin-up seems most remarkable) and therefore introduce an equal number $N$ of positive and negative vortices (per unit area), each of strength $\pm e$.

The coordinates $x_{i}$ and $y_{i}$ of the vortices are conjugate variables and the phase space is the area $A$ of the 2D container. The most probable distribution for such a system was calculated by Joyce and Montgomery[9] and follows the usual Boltzmann formula $n_{ \pm}(x, y) \propto \exp (\mp e \beta \psi(x, y))$. Consequently, if we re-scale the stream function, $e \beta \psi \rightarrow \psi$, these equilibria satisfy (from $\mathrm{Eq}(3)$ ) 


$$
\nabla^{2} \psi+\frac{\lambda^{2}}{2}\left[\frac{\exp (\psi)}{<\exp (\psi)>}-\frac{\exp (-\psi)}{<\exp (-\psi)>}\right]=0,
$$

where $\lambda^{2} / 2=-N e^{2} \beta$ and $\langle$. $>$ denotes the average over the 2-D domain.

The terms in parenthesis are the densities of negative and positive vortices respectively; the explicit introduction of the averaged values in the denominators is an important feature that ensures that different solutions of Eq (5) always represent equilibria with the same number of vortices. The boundary condition is $\psi=$ constant, but as a constant added to $\psi$ has no effect we can set $\psi=\psi_{b}=0$ on the boundary without any loss of generality.

Before discussing Eq (5) we note that by writing $\hat{\psi}=\psi-\log \left(a_{+} / a_{-}\right)^{1 / 2}$, where $a_{ \pm}=<\exp ( \pm \psi)>$, it can be reduced to the well known "sinh-Poisson" equation

$$
\nabla^{2} \hat{\psi}+\frac{\lambda^{2}}{\left(a_{+} a_{-}\right)^{1 / 2}} \sinh \hat{\psi}=0
$$

However, the boundary condition is then $\hat{\psi}=\log \left(a_{+} / a_{-}\right)^{1 / 2}$, which is unknown a priori, and cannot be set to zero as Eq (6) does not allow an arbitrary constant to be added to $\psi$. Consequently, Eq (6) with $\psi_{b}=0$, (the "sinh-Poisson equilibria" [16]), yields only equilibria that satisfy $\langle\exp (-\psi)\rangle=\langle\exp (\psi)\rangle$, e.g. those that are periodic or anti-symmetric in $x$ or $y$.

Returning to Eq (5); in general its solutions fall into two distinct classes, distinguished by whether the vorticity $\nabla^{2} \psi$, is, or is not, zero at the boundary. For both classes $\lambda^{2}$ is positive and the "inverse temperature" $\beta$ is therefore negative. It is informative to illustrate these two classes when the energy is small. Then Eq (5) reduces to

$$
\nabla^{2} \psi+\lambda^{2}(\psi-<\psi>)=0
$$

and in this limit the two classes can be distinguished by whether or not $\langle\psi\rangle$ is zero. (Of course, in the linear approximation the amplitude of $\psi$ is undetermined and $\lambda$ is a numerical eigenvalue: in general Eq (5) also determines the amplitude - and $\lambda$ can be regarded as a function of this amplitude, as measured e.g. by the energy.)

$\mathrm{Eq}(7)$ also describes the linear approximation to the statistical model of Robert and Sommeria[12] and its solutions were investigated by Chavenis and Sommeria [17]. In a rectangular domain $0 \leq x \leq a, \quad 0 \leq y \leq b$ the first class $(<\psi>=0)$ are the eigenfunctions

$$
\psi=A_{m, n} \sin (m \pi x / a) \sin (n \pi y / b)
$$

with $\lambda^{2}=\pi^{2}\left(m^{2} / a^{2}+n^{2} / b^{2}\right)$. where either $m$ or $n$ is even.

Examples of the second class $(<\psi>\neq 0)$ were computed numerically in[17]. However, they can be obtained analytically as

$$
\psi=<\psi>\left(\frac{16 \lambda^{2}}{\pi^{2}} \sum_{m, n} \frac{\sin (m \pi x / a) \sin (n \pi y / b)}{m n\left(\lambda^{2}-m^{2} \pi^{2} / a^{2}-n^{2} \pi^{2} / b^{2}\right)}\right),
$$


where both $m$ and $n$ are odd and $\lambda$ satisfies

$$
\frac{64 \lambda^{2}}{\pi^{4}} \sum_{m, n} \frac{1}{m^{2} n^{2}\left(\lambda^{2}-m^{2} \pi^{2} / a^{2}-n^{2} \pi^{2} / b^{2}\right)}=1 .
$$

In Fig(1) we show $\hat{\lambda}^{2}=\lambda^{2} A$ for the two types of solution as a function of the aspect ratio $b / a$. As noted by Chavenis and Sommeria, when $b / a<1.12, \hat{\lambda}$ is smaller for a Type II solution than for a solution of Type I.

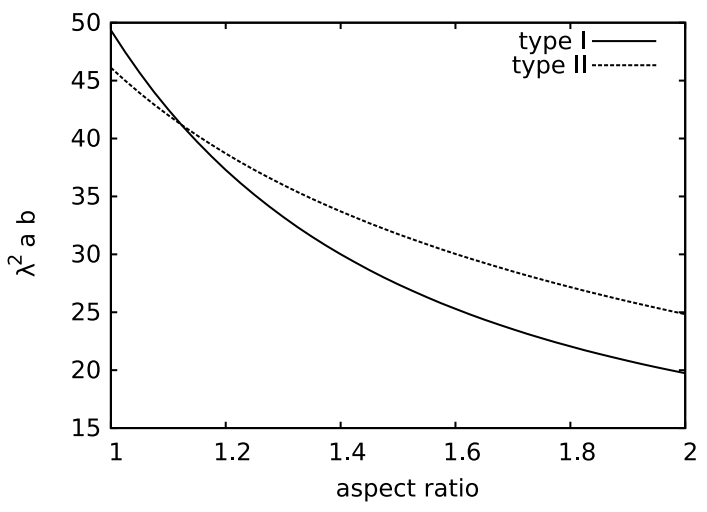

Figure 1: Effect of Aspect Ratio.

An important physical distinction between the two types of solution, which has not hitherto received attention, is their angular momentum $L_{z}=\int(x v-y u) d x d y$. Normalized to the momentum $L_{R}$ which the fluid would have if it rotated as a rigid body with the same energy, this becomes

$$
\hat{L}=\frac{L}{L_{R}}=\frac{4 \sqrt{3}}{\hat{\lambda}^{1 / 2}}\left[\left(\frac{a b}{a^{2}+b^{2}}\right)\left(\frac{<\psi>^{2}}{<\psi^{2}>-<\psi>^{2}}\right)\right]^{1 / 2}
$$

Thus we see that equilibria of the first type $(\langle\psi\rangle=0)$ always have zero angular momentum. However, equilibria of the second type have non-zero angular momentum, even though their vorticity is zero. Therefore we may refer to Type I solutions as "zeromomentum" states and to Type II as "non-zero momentum" or "spin-up" states.

Each equilibrium is a local maximum of entropy

$$
S=-\sum_{ \pm} n_{ \pm} \log \left(n_{ \pm}\right)=2 \beta E+\mu N
$$

where $\mu=\log \left(a_{+} a_{-}\right)$. In the linear approximation $S=\beta E=-\lambda^{2} E /\left(2 N e^{2}\right)$, so that $\lambda$ provides a direct measure of the relative probability of different equilibria of the same energy, with the most probable being that with smallest $\lambda$. Hence Fig (1) implies that in a square, or low aspect ratio rectangle, 2-D turbulence is more likely to evolve to a spin-up state than to a zero-momentum state. 
We can estimate the magnitude of the angular momentum generated in spin up from $\mathrm{Eq}(11)$. The simulations in $[1,2]$ which prompted the present work, were in a square box. In this case, with $\psi$ given by $\mathrm{Eq}(9)$, the normalized angular momentum predicted by the present theory is $\hat{L}_{z}=0.64$. This is significantly greater than the values $(\hat{L}=0.14-0.22)$ found in the earliest simulations[1] at Reynolds numbers $R_{e}=1-2 \times 10^{3}$, but is in good agreement with the results $(\hat{L}=0.4-0.67)$ from later simulations[2] at higher Reynolds numbers $R_{e}=1-2 \times 10^{4}$

We have computed non-linear solutions of $\mathrm{Eq}(5)$ using a relaxation method on a rectangular grid of $300 \times 300$ points. For these solutions the entropy $S$ is no longer simply proportional to $\lambda^{2} E$ and we have calculated it directly from Eq(12). Fig (2) shows, in arbitrary units, the entropy as a function of the dimensionless energy

$$
\hat{E}=\frac{E}{4 N^{2} e^{2}}=\frac{<|\nabla \psi|^{2}>}{2 \lambda^{4}}
$$

for solutions in a square boundary. (Note that we plot $-S / \hat{E}$ so that, as in Fig (1), lower data points represent higher probability.)

As already noted, in the linear approximation the spin-up state is more probable than the zero-momentum state of the same energy. Fig (2) shows that this continues to be the case for non-linear solutions, indeed the probability of the spin-up state relative to the zero-momentum state increases as $\hat{E}$ increases.

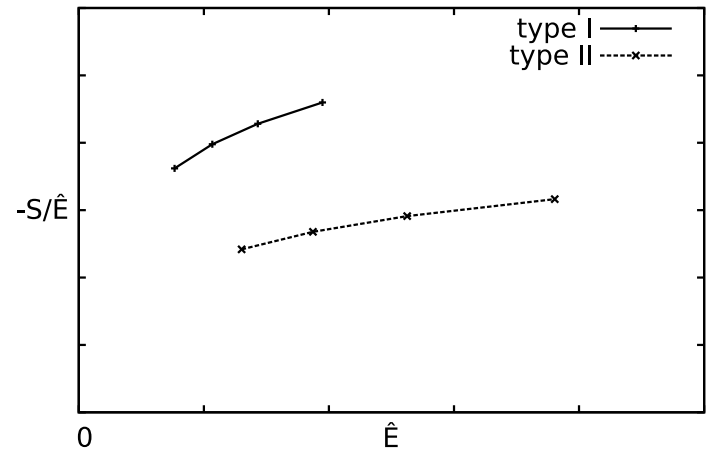

Figure 2: Entropy vs Energy. Square Boundary

This trend is also demonstrated in Figs $(3,4)$ which show results for equilibria in rectangular boundaries of aspect ratio 1.2 and 1.25. At these aspect ratios the zeromomentum state is the more probable in the linear approximation, but as $\hat{E}$ increases this situation is reversed and the spin-up state becomes the more probable.

It should be noted that each solution of (5) describes a family of point vortex equilibria and their corresponding turbulent states, - which are selected by the value ascribed to $N e$ (the total vorticity of each sign). For each solution the energy $E$ of the turbulent fluid is given by $\mathrm{Eq}(13)$ and its enstrophy $\Omega$ by

$$
\frac{\Omega}{4 N^{2} e^{2}}=\frac{<\left(\nabla^{2} \psi\right)^{2}>}{2 \lambda^{4}}
$$




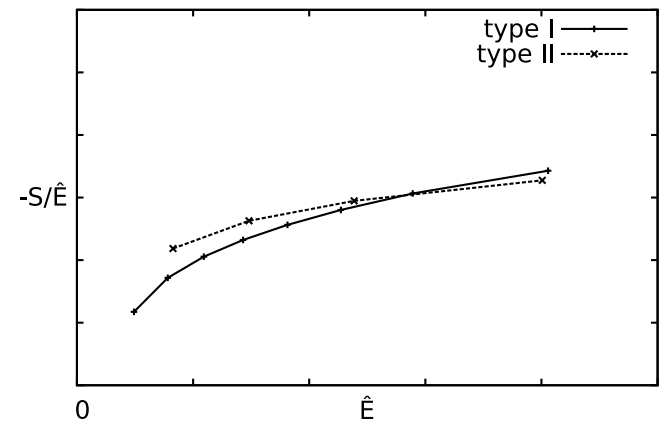

Figure 3: Entropy vs Energy. Rectangular Boundary. Aspect Ratio $=1.20$

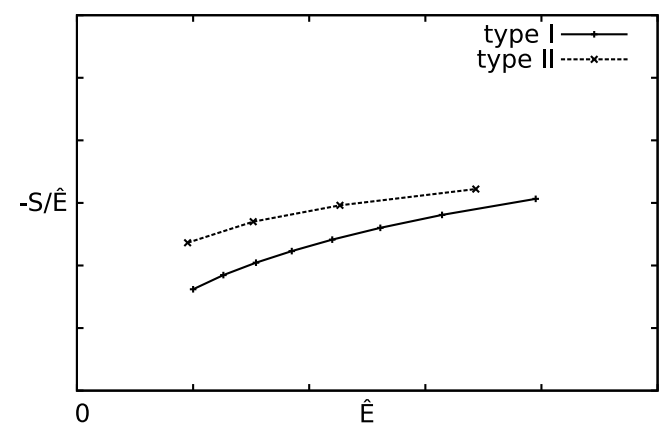

Figure 4: Entropy vs Energy. Rectangular Boundary. Aspect Ratio $=1.25$

Consequently a single solution $\psi(x, y)$ represents all turbulent states that have a particular value of $\Omega / E$. The linear solutions represent states with the minimum $\Omega / E$ - the so-called "minimum enstrophy" states $[18,19]$. The non-linear solutions represent states of larger $\Omega / E$, but they also represent states with higher energy for a given vorticity - because enstrophy rises more rapidly than energy as the non-linear terms in Eq(5) become significant.

A possible scenario for $2 \mathrm{D}$ turbulence is that initially it evolves rapidly at essentially constant energy, in accord with the point vortex model, to a state whose mean profile is given by a non-linear solution of $\mathrm{Eq}(5)$. (Of course, this state would have the same energy whether it be of Type I or Type II. However, because viscosity has a strong effect on enstrophy, and the "path" taken in the two cases is different, enstrophy is not the same in the two cases.) Later, as the enstrophy is further reduced by viscosity, the turbulence evolves through states of decreasing $\Omega / E$, represented by solutions of decreasing non-linearity, until it reaches a minimum-enstrophy state represented by a linear solution. Of course, in reality the stages merge into one another and, as $\mathrm{Eq}(5)$ does not describe a true thermodynamic limit [10,20], large deviations from the mean behavior must be expected.

Although our main concern has been to explain spin up in a rectangular boundary, as 
observed by Clercx et al [1], there have been similar direct simulations of 2D turbulence in a circular boundary $[2,21,22]$. We therefore note that in a circular boundary, Type I and Type II solutions of $\mathrm{Eq}(7)$ are degenerate $(\lambda a=3.83)$ and the zero-momentum and spin-up states therefore have equal probability at low energy.

When the non-linearity is small, $\lambda^{2}$ can be calculated by perturbation theory. This shows that, for any configuration,

$$
\frac{\Delta \lambda^{2}}{\Delta \hat{E}}=-\lambda^{2}\left(\frac{<\chi^{4}>-3<\chi^{2}>^{2}}{<\chi^{2}>^{2}}\right)
$$

where $\chi=(\psi-\langle\psi\rangle)$ and $\psi$ is the linear solution. Applied to the circular boundary case, this confirms that non-linearity splits the degeneracy of the two solutions and that the spin-up state becomes more probable than the zero-momentum state.

In fact, however, the circular boundary is unique in that angular momentum is an exact invariant of the vortex/rod model (since any force is normal to the boundary) and the spin-up state is inaccessible from an initial state of zero angular momentum. (In the numerical simulations there are two forces on the boundary - the pressure, which also does not affect angular momentum, and a viscous drag from the "no-slip" boundary condition. This drag, which is not represented in the vortex/rod model, and is small at high Reynolds number, can affect the angular momentum.)

In conclusion, although at first sight the phenomenon of "spin-up" (the spontaneous acquisition of angular momentum) in simulations of 2D turbulence may appear surprising, it is in fact a natural feature of statistical models of 2-D turbulence. When the circulation is zero there are two types of statistical equilibrium in a bounded domain. One is the well known sinh-Poisson equilibrium which carries no angular momentum: the other type has non-zero angular momentum even though its total vorticity and circulation are zero.

The equilibria we have calculated have similar properties to the states seen in direct numerical simulations of $2 \mathrm{D}$ turbulence. At a fixed energy the relative probability of the two types depends weakly on the energy and strongly on the shape of the container. In addition the angular momentum predicted for the spin up states agrees well with that found in simulations at high Reynolds number, but less well with that found at lower Reynolds number - where viscosity plays a larger role.

\section{References}

[1] H.J.H Clercx, S.R. Maassen and G.J.F. van Heijst, Phys Rev. Lett. 80, 5129 (1998)

[2] H.J.H Clercx, A.H. Nielsen, D.J. Torres and E.A. Coutsias, Eur. J. Mech. B20, 557 (2001)

[3] T.S. Lundgren and Y.B. Pointin, J. Stat Phys. 17, 323 (1977)

[4] Y.B. Pointin and T.S. Lundgren, Phys. Fluids 19, 1459 (1976) 
[5] R.H.Kraichnan and D. Montgomery, Rep. Prog. Phys. 43, 547 (1979)

[6] D. Montgomery and G. Joyce, Phys. Fluids 17, 1139 (1974)

[7] L. Onsager, Nuovo Cimento Suppl. 6, 279 (1947)

[8] J.B. Taylor and B.McNamara, Phys. Fluids 14, 1492 (1971)

[9] G. Joyce and D. Montgomery, J. Plasma Phys. 10, 107 (1973)

[10] S.F. Edwards and J.B. Taylor, Proc. Roy. Soc. A336, 257 (1974)

[11] C.C. Lin, Proc.Nat. Acad. Sci. USA 27, 570 (1941)

[12] R. Robert and J. Sommeria, J. Fluid Mech. 229, 291 (1991)

[13] D. Lynden-Bell, Mon. Not. R. Astro. Soc. 136, 101 (1967)

[14] W.H. Schubert et. al., J.Atmos. Sci. 56, 1197 (1999)

[15] F. Bouchet and J. Sommeria, J. Fluid Mech. 464, 165 (2002)

[16] D. Book, S. Fisher and B.E. McDonald, Phys. Rev. Lett. 34, 4 (1975)

[17] P.H. Chavenis and J. Sommeria, J. Fluid Mech. 314, 267 (1996)

[18] C.E. Leith, Phys. Fluids 27, 1388 (1984)

[19] F.P. Bretherton and D.B. Haidvogel, J. Fluid Mech. 78,129 (1976)

[20] J Frohlich and D Ruelle, Commun. Math. Phys. 87, 1 (1982)

[21] K. Schneider and M. Farge, Phys. Rev. Lett. 95, 244502 (2005)

[22] S.Li and D. Montgomery, Phys. Lett. A218, 281 (1996) 